\title{
Amiloidose de conjuntivas palpebrais inferiores: um relato de caso
}

Lower eyelid conjunctival amyloidosis: a case report

Bruno Hirt ${ }^{1}$ (D), Marcos Bortoluzzi Worma² (), Graziela Junges Crescente Rastelli ${ }^{3}$ (), Rodrigo Beraldi Kormann ${ }^{4}$ (1)

${ }^{1}$ Curso de Especialização em Oftalmologia, Hospital de Olhos do Paraná, Curitiba, PR, Brasil. ${ }^{2}$ Curso de Graduação em Medicina, Universidade Positivo Curitiba, PR, Brasil. ${ }^{3}$ Departamento de Medicina, Faculdade Evangélica Mackenzie do Paraná, Curitiba, PR, Brasil. ${ }^{4}$ Departamento de Oftalmologia, Hospital de Olhos do Paraná, Curitiba, PR, Brasil.

Hirt B, Worma MB, Rastelli GJ, Kormann RB. Amiloidose de conjuntivas palpebrais inferiores: um relato de caso. Rev Bras Oftalmol. 2021;80(4):e0019.

https://doi.org/10.37039/1982.8551.2021001

Descritores:

Amiloidose; Pálpebras; Olho;

Conjuntiva

Keywords:

Amyloidosis; Eyelids; Eye;

Conjunctiva

Recebido: 08/07/2020

Aceito:

12/4/2021

Autor correspondente: Bruno Hirt Rua Prof. Pedro Viriato Parigot de Souza, 3155, Campo Comprido, Curitiba, PR, 81200-452, Brasil.

E-mail: brunohirt.med@gmail.com.

Instituição:

Hospital de Olhos do Paraná.

Fonte de auxílio à pesquisa: não financiado.

Conflitos de interesse: os autores declaram que não há conflitos de interesses.

\section{RESUMO}

A amiloidose é caracterizada pela deposição extracelular de fibrilas amiloides proteicas nos tecidos, incluindo conjuntiva, motivada por sua insolubilidade em proteínas proteolíticas. A amiloidose conjuntival, afetando especificamente a conjuntiva palpebral, é um achado raro na prática clínica. Os autores descrevem um caso de um paciente do sexo masculino, caucasiano, de 71 anos, apresentando lesões bilaterais localizadas na conjuntiva da pálpebra inferior, elevadas, bem delimitadas, amareladas, aliadas a pálpebras desestabilizadas. Foi realizada excisão cirúrgica das lesões, e o exame histológico confirmou se tratar de amiloidose conjuntival. Não houve recorrência após o procedimento. A amiloidose conjuntival afetando ambas as pálpebras inferiores é um achado raro na literatura. 0 manejo da amiloidose conjuntival depende da extensão do envolvimento local e do status sistêmico do paciente, variando de sintomático, com o uso de lubrificantes, até cirúrgico, com a remoção das lesões.

\section{ABSTRACT}

Amyloidosis is characterized by extracellular deposition of protein amyloid fibrils in tissues, including conjunctiva, which are insoluble in proteolytic proteins. Conjunctival amyloidosis specifically in the palpebral conjunctiva is a rare finding in clinical practice. The authors described the case of a 71-yearold caucasian male patient, with bilateral lesions located in the lower eyelid conjunctiva, elevated, welldefined, yellowish, combined with destabilized eyelids. Surgical excision of the lesions was performed, and the histological examination confirmed conjunctival amyloidosis. There was no recurrence after the procedure. Conjunctival amyloidosis in both lower eyelids is a rare finding in the literature. The management of conjunctival amyloidosis depends on the extent of local involvement and patient's general health status, varying from symptomatic, with use of ocular lubricants, to surgical, by removing the lesions. 


\section{INTRODUÇÃO}

A amiloidose é um distúrbio sistêmico, caracterizado pela deposição extracelular de fibrilas amiloides proteicas em vários tecidos, incluindo pálpebras e conjuntiva, motivada pela insolubilidade de proteínas proteolíticas, com acúmulos teciduais e efeitos de citotoxicidade, que podem prejudicar a estrutura tecidual e a função do órgão afetado. Essas fibrilas são consideradas anormais, rígidas e sem ramificações, ligando-se facilmente a corantes utilizados em análises histopatológicas..$^{(1-3)}$

A amiloidose pode ser classificada em três tipos principais, conhecidos como primário, secundário e sistêmico. ${ }^{(2)} \mathrm{A}$ amiloidose conjuntival raramente é descrita como associada a envolvimento sistêmico. ${ }^{(2-5)} \mathrm{A}$ amiloidose primária, conhecida como idiopática e sem particularidades clínicas, é uma condição incomum que afeta a pálpebra inferior, considerada um achado raro na prática clínica e na literatura. ${ }^{(1,6,7)}$

O envolvimento orbital e/ou periocular pode apresentar graus variados de comprometimento, dependendo do tamanho e da localização das massas, com lesões semelhantes a pápulas fusiformes ou polipoides amareladas, como massas extraoculares ou subconjuntivais, ou como infiltração difusa de tecidos, ambas podendo causar diplopia, dor, ptose mecânica, desvio do olho e mobilidade ocular reduzida. $(3,5,6)$

Os autores descrevem um caso de amiloidose conjuntival bilateral, relatando sintomas, diagnóstico e tratamento. A coleta e a avaliação de informações protegidas de saúde do paciente neste estudo aderiram aos princípios da Declaração de Helsinque. O presente estudo foi aprovado na Plataforma Brasil, CAAE : 33649820.2.0000.0103.

\section{RELATO DO CASO}

Paciente do sexo masculino, 71 anos, caucasiano, ex-bancário, diabético e cardiopata, compareceu a uma consulta oftalmológica no Hospital de Olhos do Paraná, em maio de 2019, relatando o aparecimento de lesões com aumento progressivo em ambas as pálpebras inferiores nos 4 meses anteriores a consulta. O paciente apresentava sintomas de superfície ocular, como hiperemia, sensação de corpo estranho e queimação.

O exame externo mostrou três lesões lobulares, de superfície lisa, localizadas na conjuntiva palpebral inferior bilateralmente - mais especificamente uma lesão em conjuntiva tarsal, envolvendo também fórnice temporal à direita, e duas lesões, uma em conjuntiva tarsal central e outra temporal, envolvendo ambos fórnice e conjuntiva tarsal à esquerda. As lesões se apresentavam elevadas, bem definidas, amareladas (Figura 1), justificando os sintomas do paciente, visto que elas impediam o contato correto entre a conjuntiva palpebral e a bulbar. Ao exame, o tempo de quebra do filme lacrimal estava reduzido e havia ceratite punctata em ambos os olhos. As pálpebras se mostraram desestabilizadas bilateralmente, ora com tendência para entrópio, ora para ectrópio.

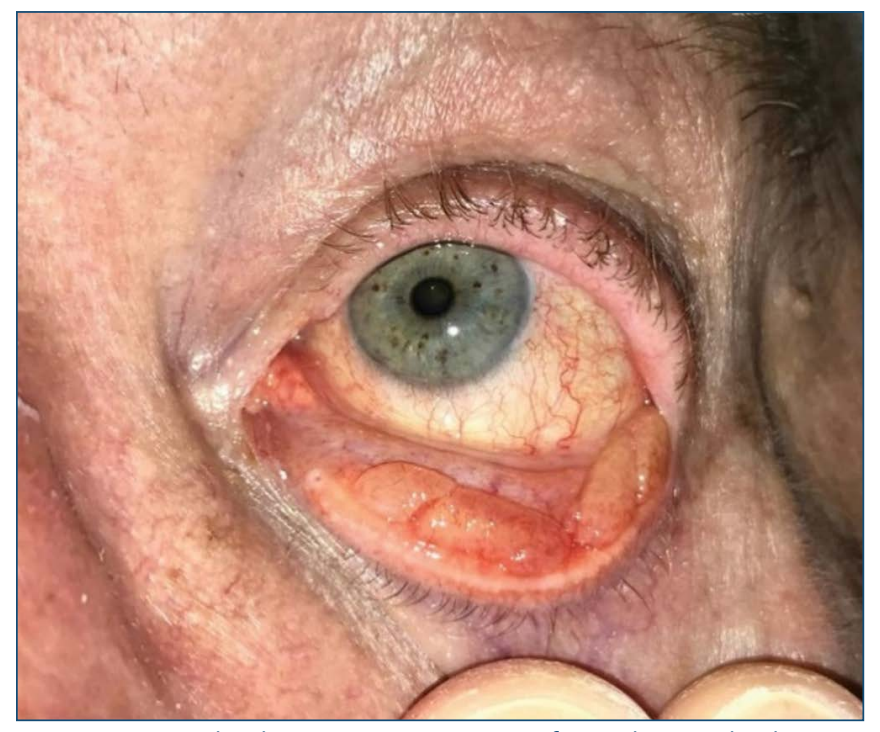

Figura 1. Amiloidose em conjuntiva afetando a pálpebra inferior esquerda.

Optou-se por sedação e remoção das lesões, com envio delas para avaliação anatomopatológica. Após a excisão, as lesões foram acondicionadas em recipiente contendo formalina $10 \%$ e enviadas para exame histológico. Ao exame macroscópico, duas lesões, uma medindo $10 \mathrm{mmx} \times \mathrm{mm} \times 3 \mathrm{~mm}$ e a outra $3 \mathrm{~mm} \times 3 \mathrm{~mm} \times 3 \mathrm{~mm}$, foram identificadas e processadas para exame, tendo sido embebidas em parafina e coradas por hematoxilina-eosina. Para confirmar a presença de material amiloide, utilizou-se a coloração cristal violeta. A análise das lâminas histológicas revelou segmentos de mucosa conjuntival de cor acastanhada, contendo depósito de material amiloide denso, caracterizados como amiloidose conjuntival (Figura 2).

Os retornos para avaliação pós-operatória se deram no primeiro, sétimo e trigésimo dia e 1 ano após a cirurgia. Não houve recidiva e nem outros sintomas no período (Figura 3).

A avaliação sistêmica do paciente não mostrou alterações dignas de nota, não havendo indício de amiloidose extraocular. Desta forma, concluiu-se se tratar de amiloidose conjuntival primária. 


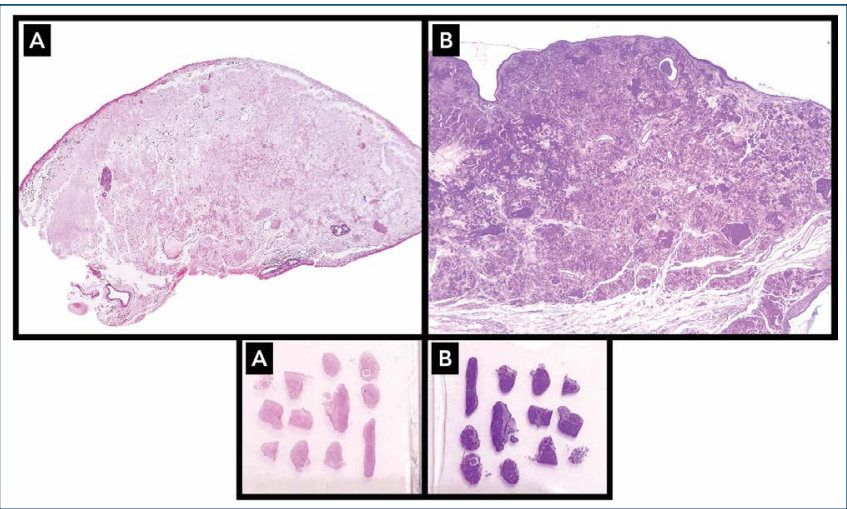

Figura 2. (A) Cortes histológicos mostrando fragmento de conjuntiva contendo denso depósito de material amiloide (hematoxilina-eosina, 40X). (B) Coloração especial cristal violeta facilita a visualização dos depósitos amiloides (cristal violeta, 100X).
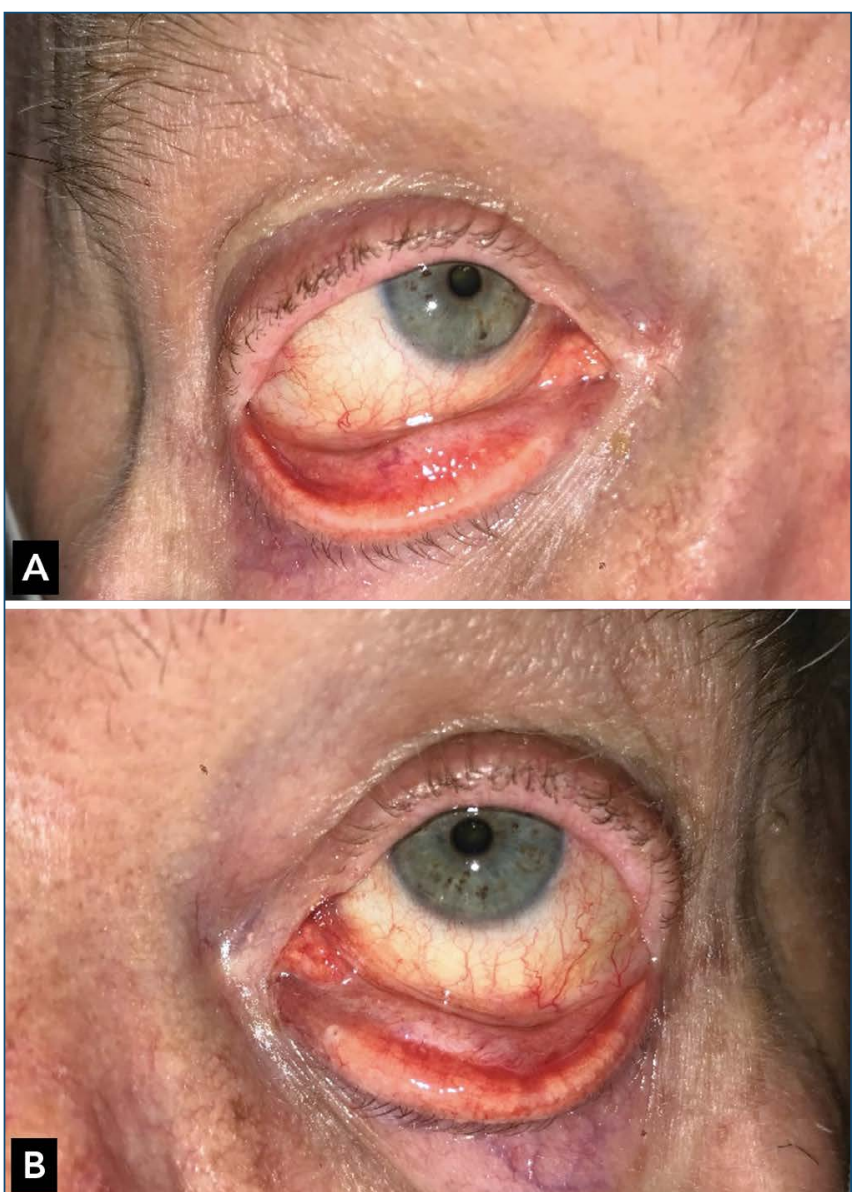

Figura 3. Foto de 1 ano pós-operatório do olho direito $(A)$ e do olho esquerdo (B). Nota-se ausência de recidivas.

\section{DISCUSSÃO}

Os autores relatam um caso de amiloidose conjuntival em um paciente do sexo masculino, idoso, que apresentou acometimento conjuntival inferior bilateral, com sintomas de superfície ocular, tempo de ruptura do filme lacrimal reduzido e presença de lesão em conjuntiva tarsal e fórnice à direita, além de duas lesões em conjuntiva tarsal, uma delas também em fórnice, à esquerda. O método de tratamento de escolha foi a excisão cirúrgica das lesões, com posterior análise anatomopatológica.

No caso apresentado, o acometimento foi bilateral e envolveu conjuntiva tarsal inferior direita e esquerda com acometimento simultâneo de fórnices. Outros poucos casos bilaterais também foram relatados na literatura: um paciente de 30 anos com acometimento de conjuntiva tarsal e fórnice bilateralmente; ${ }^{(3)}$ uma paciente de 66 anos com acometimento conjuntival em fórnices bilateralmente; ${ }^{(4)}$ um paciente de 59 anos com acometimento de conjuntiva tarsal bilateral; (5) uma paciente de 69 anos com acometimento de fórnice inferior bilateral, ${ }^{(6)}$ um paciente de 43 anos com acometimento de conjuntiva palpebral bilateral;:(8) uma paciente de 48 anos com acometimento de conjuntiva palpebral superior e inferior bilateral; ${ }^{(9)}$ uma paciente de 77 anos com acometimento de conjuntiva palpebral inferior bilateral; ${ }^{(10)}$ três pacientes com idade média de 60 anos com acometimento conjuntival bilateral:;11) e, finalmente, uma paciente de 39 anos com acometimento conjuntival em fórnice inferior bilateral. ${ }^{(12)}$

A maioria dos relatos e séries de casos na literatura trazem dados de acometimento unilateral, cujos pacientes, em sua maioria adultos de meia-idade, apresentavam comumente envolvimento de conjuntiva palpebral e/ou fórnices. . $2,5-7,9-11)$ Uma série de 14 casos de amiloidose diagnosticados de 1994 a 2014 foi descrita em conjunto com outros 69 casos da literatura (de 2004 a 2015), mostrando acometimento por amiloidose de alguma estrutura ocular, sendo que $47(68,1 \%)$ pacientes tinham acometimento conjuntival. Destes pacientes, 36 (76,6\%) apresentaram acometimento conjuntival unilateral, sendo que $22(61,1 \%)$ evidenciaram lesão em conjuntiva palpebral, 19 (52,8\%) em conjuntiva bulbar e quatro $(11,1 \%)$ em fórnice. ${ }^{(5)}$ Tais fatos corroboram a infrequência de casos unilaterais, ao mesmo tempo que evidenciam a raridade de um caso bilateral.

O envolvimento sistêmico pela amiloidose é possível devido à disfunção tecidual/do órgão causada pela deposição de proteínas amiloides e sua citotoxicidade. Além disso, menos de 10\% dos casos apresentam apenas sintomas localizados, também chamado de amiloidose primária. ${ }^{(7)}$ No caso do estudo, o paciente possivelmente entra nessa classificação, pois não apresentava outras alterações oculares, e os exames sistêmicos realizados não mostraram alterações suspeitas de amiloidose em outras localizações, corroborando a raridade do caso.

Em relação ao acometimento conjuntival, a deposição de proteína amiloide pode ou não ser sintomática, 
geralmente ocorrendo edema unilateral, irritação e hemorragia subconjuntival, e, dependendo da localização e da área afetada, o paciente pode ainda apresentar dor, equimose e ptose. ${ }^{(6,8)} \mathrm{O}$ paciente relatado apresentou lesões localizadas na conjuntiva tarsal palpebral, e os principais locais de acometimento tendem a ser a conjuntiva palpebral, a conjuntiva bulbar e os fórnices. Outros sítios de acometimento são os músculos extraoculares e as glândulas lacrimais, por exemplo. ${ }^{(8,9)}$

No presente relato, pelo fato de as lesões estarem localizadas na conjuntiva tarsal palpebral e forniceal, a apresentação clínica do paciente estava restrita a sintomas óculo-palpebrais, como irritação conjuntival, desconforto devido ao deslocamento palpebral causado pelas lesões e desestabilização palpebral pelo mesmo motivo, ora gerando entrópio, ora ectrópio.

O tratamento do paciente baseou-se na biópsia excisional das lesões conjuntivais. A análise histológica das peças cirúrgicas mostrou o diagnóstico preciso, uma vez que a identificação clínica dessa doença pode ser dificultada por sua raridade e pela variedade de sintomas. O manejo da amiloidose conjuntival depende da extensão do envolvimento local e do status sistêmico do paciente. . $3,5,7)^{\mid 3,7}$ A excisão cirúrgica e o exame histopatológico de lesões únicas ou difusas, além de serem considerados as melhores abordagem, podem aliviar os sintomas causados pela massa de depósitos amiloides, ${ }^{(2,10,11)}$ embora ainda não exista consenso de manejo, uma vez que a discussão sobre tratamento agressivo e/ou conservador ainda está por ser resolvida. ${ }^{(4)}$ Em alguns casos, enquanto se aguarda a cirurgia ou em caso de dúvida se o procedimento é necessário, o controle temporário dos sintomas com lubrificantes oculares também se mostrou útil. ${ }^{(6,2)}$

Os principais diagnósticos diferenciais relacionados à amiloidose conjuntival incluem carcinoma de célula basal ou escamosa, leucemia e linfoma. ${ }^{(3,6,6,710)} \mathrm{O}$ aspecto róseo da amiloidose é similar ao encontrado no linfoma ou na leucemia; mas o acometimento conjuntival palpebral e a presença de hemorragia local tendem a descartar essas possibilidades. Sob outra perspectiva, carcinoma de célula basal ou escamosa são mais ligados a pacientes com história prévia de neoplasia. ${ }^{(3,6,10)}$ Todas essas informações devem ser levadas em consideração ao se examinar um paciente com massa conjuntival suspeita, visto que a amiloidose é uma doença rara e pode ser diagnosticada incorretamente.

É importante reconhecer a conjuntiva como um local em que a amiloidose pode ocorrer. O curso da amiloidose primária é geralmente benigno. Ademais, deve-se considerar que a lesão pode fazer parte de afecção sistêmica, realizando-se a pesquisa de focos extraoculares de amiloidose, plausíveis de potencial dano à saúde geral do paciente. ${ }^{(4,5,10)}$

\section{REFERÊNCIAS}

1. Brownstein $\mathrm{MH}$, Elliott $\mathrm{R}$, Helwig EB. Ophthalmologic aspects of amyloidosis. Am J Ophthalmol. 1970;69(3):423-30.

2. Medel Jiménez R, Sánchez España JC, Vasquez LM, Tapia Bahamondes A, Rondón M, Francesc T, et al. Orbital and peri-orbital amyloidosis: a report of four cases. Orbit. 2019;38(2):148-53.

3. Caggiati A, Campanella A, Tenna S, Cogliandro A, Potenza C, Persichetti P. Primary amyloidosis of the eyelid: a case report. In Vivo. 2010;24(4):575-8.

4. Correa LJ, Maccio JP, Esposito E, Monti R, Gonzalez-Castellanos ME, Paradelo $\mathrm{M}$, et al. Systemic amyloidosis with bilateral conjunctival involvement: a case report. BMC Ophthalmol. 2015;15(1):77.

5. Mora-Horna ER, Rojas-Padilla R, López VG, Guzmán MJ, Ceriotto A Salcedo G. Ocular adnexal and orbital amyloidosis: a case series and literature review. Int Ophthalmol. 2016;36(2):281-98

6. Demirci H, Shields CL, Eagle RC Jr, Shields JA. Conjunctival amyloidosis: report of six cases and review of the literature. Surv Ophthalmol. 2006;51(4):419-33.

7. Shields JA, Eagle RC Jr, Shields CL, Green M, Singh AD. Systemic amyloidosis presenting as a mass of the conjunctival semilunar fold. Am J Ophthalmol. 2000;130(4):523-5.

8. Chaturvedi P, Lala M, Desai S, Sanghvi V. A rare case of both eyelids swelling: isolated conjunctival amyloidosis. Indian J Ophthalmol. 2000;48(1):56-7

9. Hamill EB, Thyparampil PJ, Yen MT. Localized immunoglobulin light chain amyloid of the conjunctiva confirmed by mass spectrometry without evidence of systemic disease. Ophthal Plast Reconstr Surg. 2017;33(5):e108-10.

10. Suesskind D, Ziemssen F, Rohrbach JM. Conjunctival amyloidosisclinical and histopathologic features. Graefes Arch Clin Exp Ophthalmol. 2015;253(8):1377-83.

11. Leibovitch I, Selva D, Goldberg RA, Sullivan TJ, Saeed P, Davis G, et al. Periocular and orbital amyloidosis: clinical characteristics, management, and outcome. Ophthalmology. 2006;113(9):1657-64.

12. Lee HM, Naor J, DeAngelis D, Rootman DS. Primary localized conjunctival amyloidosis presenting with recurrence of subconjunctival hemorrhage. Am J Ophthalmol. 2000;129(2):245-7. 